did met difter in recosnition when buth target and distractor words were presented together during study. Since Ss had an opportunity to compare the target and districtor actively during study, they could have attended to those word features (semantic, phonetic, associative, visual) that served to make synonyms and homophones as discriminable as two unrelated words.

RHII:RI:NCIS

tDAlls. J. A. Human memory. New York: Heviraw-lill. 1967

ANISt 11.1). M.. \& KNAPP. M. Association. synonymity, and dirctionality in talso recognition. Journal of lexperimental Pyychology. 1968.77, 171-179.

BLiSClikE H.. \& LINON, R. Encoding homophones and synonyms for verbal diximination and recosnition. Psychonomic Science. 1969, 14, 269-270.

MARSHALL, J. I.. ROLSI: R. O.. JR.. \& TARPY. R. M. Acoustic versus associative models of short-term memory coding. Psychonomic Science. 1969. 14. 54-55.

NORMAN, D. A. Memory and attention in introduction to human information processing. New York: Wiley. 1969. Chap. 7.
JHORNDIKL I. L.. \& LORG; . I The teacher handhook of 30.000 words. New rork Bureau of Publications. [eachers college. Columbia L niversity. 1944.

L'NDFRWOOD. B. J.. \& I RILND, J. S. Errors in recognition learning and retention. Journal of Ixperimental Psychology, 1968, 78. 55-63. NOTFS

1. This investigation was supported by USPHS Research Grant MH-08556 and USPHS Research Scientist Award K3-MH-23.796 to Herman Buschke from the National Institute of Menta Health. The assistance of Kirk Gibson in programming and the comments of Howard Ranken are gratefully acknowledged.

2. USPHS Postdoctoral Fellow, Fellowship No. 1-l 02-HD-29.231-0I.

3. Now at the Saul R. Korey Department of Neurology, Albert Einstein College of Medicine, Bronx. New York 10461 .

4. The correction formula employed was standard one: $[\mathrm{P}(\mathrm{C})-\mathrm{g}] /(\mathbf{l}-\mathrm{g})$, where $\mathrm{P}(\mathrm{C})$ is the observed proportion correct and $\mathrm{g}$ is the probability of a correct guess, 0.5 in this case.

5 . It is possible that the words comprising many $S$ pairs were not completely synonymous, so that the semantic content of a study word was shightly different from that of its distractor. In that case. recognition of $S$ pairs would be expected to be somewhat better than that obtained when both words of an $S$ pair are pertect synonyms.

\title{
Rated acoustic (articulatory) similarity for word pairs varying in number and ordinal position of comimon letters ${ }^{1}$
}

\begin{abstract}
DOUGLAS I. NEISON and LOUISE D. NELSON, University of South Florida, Tampa, Fla. 33620
\end{abstract}

Ratings of "similarity-in-sound" for pairs of words sharing letters in various ordinal positions were obtained using a 7-category graphic scale on which degrees of similarity were specified $b y$ verbal labels. Judged acoustic (articulatory) similarity increased as number of shared letters increased from zero to one. two. and three letters. Pairs overlapping in first letters were rated significantly more similar than pairs sharing middle or last letters. For pairs sharing letters within two ordinal positions. judged similarity increased in the order of first-and-middle, first-and-last, middle-and-last. Implications for the hypothesis that verbal stimuli are coded by pronunciation were discussed.

Manipulations of ordinal position of identical letters within sets of three-letter words have produced relatively consistent findings that have depended upon characteristics of the learning task. When discriminations between stimuli are required, as in paired-associate (Nelson \& Rowe, 1969) and serial-recall learning (Nelson, 1969), difficulty of acquisition increased in the order middle (M), last (L) first (F) for stimulus sets sharing letters within single locations and in the order middle-and-last $(\mathrm{M}+\mathrm{L})$, first-and-middle $(F+M)$, first-and-last $(F+L)$ for sets in which letters were shared within two ordinal positions. A similar ordering for the dual-locus conditions also has been reported when paired-associate stimuli were nonsense syllables (Richardson \& Chisholm, 1969; Runquist, 1968a). When stimuli could be grouped together as in free recall (Nelson, 1969) and when paired-associate stimuli shared letters with their responses (Nelson \& Garland, 1969) orders of difficulty by identity locus were reversed.

One explanation of these results assumes that the stimuli were coded by pronunciation, rendering the codes subject to acoustic (or articulatory) interference or facilitation depending upon the task requirements (Runquist, 1968a). Accordingly, to account for the effects of variations in locus of identical letters in the various learning tasks, this hypothesis predicts that rated acoustic similarity should increase in the order $\mathrm{M}, \mathrm{L}$, and $\mathrm{F}$ for pairs of words sharing letters within a single locus and in the order $M+L, F+M$, and $F+L$ for word pairs sharing letters within two ordinal positions. The only available data have been inconsistent with this prediction. Runquist (1968b) found that word pairs sharing first letters were rated as more similar than were pairs overlapping in middle or last letters, which do not differ. No apparent differences were found between pairs sharing letters within two positions. However, the method used for obtaining ratings required estimations of the percentage $(0-100)$ of similarity shared between stimuli of the pair, with the similarity attribute unspecified. To the indeterminate extent that similarity estimates were made on the basis of counting the number of common letters, as is suggested by ratings around $66 \%$ for all dual-locus pairs, differences as a function of locus would be attentuated. The purpose of the present study was to scale pairs of words overlapping in various ordinal positions with the similarity attribute specified as "sound similarity." Specification of the acoustic dimension was expected to reduce the likelihood of rating on the basis of letter counting and, perhaps, reveal greater differences within the overlap conditions. Moreover, ratings made on the basis of acoustic similarity should provide evidence relevant to the adequacy of the pronunciation hypothesis as an explanation for the ordinal-position effect.

\section{MATERIALS}

The items to be rated consisted of 108 pairs of words. Eighty-four of these pairs were generated by taking all possible pairwise comparisons within each of the following three lists: PAN, PAT, PIN, PIT, FAN, FAT, FIN, FIT; BAD, BAG, BED, BEG, LAD, LAG, LED, LEG; and HAM, HAT, HUM, HUT, RAM, RAT, RUM, RUT. This procedure produced four pairs of items within each list that shared letters in all possible positions, including zero. Thus, there were 12 pairs of words representing each of the seven overlap conditions. The 12 pairs representing complete identity were generated by selecting four single words from each list and by pairing each word with itself. These words were selected so that each different initial, medial, and terminal letter was equally represented (i.e., for the first list, the items chosen for self-pairing were PAN, PIT, FAT, FIN). The remaining 12 pairs were chosen, by E's judgment, so that four pairs represented each of the following three similarity conditions: high 
acoustic-high formal (PEA-SEA, FIR-SIR, COW-NOW, HUT-MUT), high acoustic-low formal (PEA-SKI, WHY-RYE, FLU-TOO, SEW-HOE), and low acoustic-high formal (PEA-YEA, FIR-AIR, COW-LOW, HUT-OUT).

\section{PROCEDURE}

Each $\mathbf{S}$ was handed a booklet containing the 108 pairs. On each page, a single word pair was typed in capital letters, and below this was a verbally labeled, 7.point scale indicating increasing degrees of similarity. (See Table 1.)

Ss were instructed to pronounce the words of the pair to themselves and then indicate how "similar-in-sound" the words were to each other by checking the appropriate category on the scale. The same E administered the task to all Ss and allowed 15-20 min for completion. The sequence of pairs was independently randomized for each S.

\section{SUBJECTS}

There were four groups of 23-28 Ss each. Each group consisted of an ongoing psychology course. Two groups $(\mathrm{N}=50)$ rated the pairs in one order (PAN PAT), and the other two groups $(N=46)$ rated the pairs in the reverse order (PAT PAN).

\section{RESULTS}

Numerical values of 1.7 were assigned to the seven categories, with increasingly larger values being assigned to increases in rated similarity. A mixed-model ANOVA, with order as the between-S variable and overlap condition as the within-S variable, showed that only the latter source was significant $[F(7,658)=743.83]$. Mean similarity rating increased in the order zero (1.74), L (2.60), M (2.69), F (3.33), F + M (3.99), $\mathrm{F}+\mathrm{L}$ (4.17), $\mathrm{M}+\mathrm{L}$ (5.33), and identity (6.98). Fisher's least significant difference was 0.17 , indicating that each mean was statistically different from each other mean, except for the difference between $M$ vs $L$.

Mean similarity ratings were $5.41,5.00$, 2.78 , and 1.74 , respectively, for pairs designated as high acoustic-high formal, high acoustic-low formal, low acoustic-high formal, and low acoustic-low formal (Condition 0). ANOVA indicated that acoustic $[F(1,94)=707.47]$ and formal similarity $[F(1,94)=486.22]$, and the interaction of these variables $[F(1,94)=19.38]$, were all significant. Pair order and all other interactions with this variable were not statistically reliable. The least significant difference was 0.15 , indicating that each mean was different from each other mean. On the assumption that pairs within high and low formal-similarity conditions were equally high or equally low in acoustic similarity, this pattern of means suggests that letter counting influenced the ratings. Pairs sharing letters were rated more similar than those not sharing letters. However, the direction of the interaction suggested that ratings were more affected by acoustic (articulatory) than by formal characteristics. Differences between high and low formal similarity were. less when acoustic similarity was high than when it was low.

\section{DISCUSSION}

All pairs of words sharing a letter within one or more ordinal positions are judged as being more similar in sound than pairs with no letters in common. Moreover, pairs of words sharing $F$ letters are rated as more similar than pairs overlapping in $\mathrm{M}$ or $\mathrm{L}$ letters, which do not differ. Rated similarity of words sharing letters within two ordinal positions was higher than pairs sharing single letters and less than identity pairs. Within dual-locus conditions, judged similarity increased in the order $F+M$, $F+L, M+L$. Although the findings within pairs overlapping within single positions were consistent with those reported by Runquist (1968b), results for pairs overlapping within two positions were not. The analysis of pairs differing in formal vs acoustic characteristics suggests that the apparent lack of differences that he reported may have been a result of failing to specify the similarity attribute, so that estimations may have been made more on the basis of number of common letters than on acoustic or articulatory characteristics.

The hypothesis that the ordinal-position effect can be explained by the assumption that words are coded by pronunciation and stored and/or retrieved as acoustic or articulatory codes has not been well supported by rating data. This hypothesis predicts that the higher the similarity rating given to a particular overlap condition relative to other letter-sharing conditions, the greater the interference effect when stimuli must be discriminated from each other and the greater the facilitation effect when these stimuli can be recalled together. However, consistent

\section{Table 1}

\section{PAN PAT}

$\begin{gathered}\text { completely } \\ \text { dissimilar }\end{gathered}$

differences in acquisition rates have been found for stimulus sets in which identical letters were located within $\mathrm{L}$ relative to $\mathrm{M}$ positions, even though pairs sharing letters within each of these positions are judged as equally similar. Moreover, in tasks requiring stimulus discriminations (Nelson, 1969; Nelson \& Rowe, 1969), acquisition rate has been fastest for Condition $\mathrm{M}+\mathrm{L}$ relative to Conditions $F+M$ and $F+L$, even though these stimuli have been judged as more similar in sound and, hence, should be more difficult to discriminate because of the generation of a greater degree of acoustic interference. Similarly, when stimuli could have been grouped or recalled together (Nelson, 1969; Nelson \& Garland, $1969)$ and acoustic similarity should have facilitated performance, recall for this condition was generally below that for the other dual-locus conditions. The only way to reconcile the rating and acquisition data for the $\mathrm{M}+\mathrm{L}$ condition would be to assume that different processes are involved in the two tasks. Thus, rhyming may have been a prepotent response in the rating task resulting in higher similarity ratings for pairs of words agreeing in terminal sounds. However, in order to account for the patterns of acquisition rates, it would have to be assumed that, if rhyming were eliminated, pairs sharing terminal letters would actually be rated as less similar than other dual-locus pairs. This outcome seems unlikely. Furthermore, it also seems unlikely that rhyming would operate during the rating task and not during an acquisition task, especially if it might facilitate performance as in free recall via clustering.

\section{REFERENCES}

NELSON, D. L. Information theory and stimulus encoding in free and serial recall: Ordinal position of formal similarity. Journal of Experimental Psychology, 1969, 80, 537-541. NELSON, D. L., \& GARLAND, R. M. Amount and locus of stimulus-response overlap in paired-associate acquisition. Journal of Experimental Psychology, 1969, 82, 297-300.

NELSON, D. L., \& ROWE, F. A. Information theory and stimulus encoding in paired-associate acquisition: Ordinal position of formal similarity. Joumal of Experimental Psychology, 1969, 79, 342-346.

RICHARDSON, J., \& CHISHOLM, D. C. Transfer of cue selection based on letter position. Journal of Experimental Psychology, 1969, 80, 299-303.

RUNQUIST, w. N. Formal intralist similarity in paired-associate learning. Journal of Experimental Psychology, 1968a, 78, 634-64l. RUNQUIST, W. N. Rated similarity of high $\mathrm{m}$ CVC trigrams and words and low $m$ CCC trigrams. Journal of Verbal Learning \& Verbal Behavior, 1968b, 7, 967-968. NOTE

1. This research was supported by a grant (MH 16360-01) to the first author from the National Institute of Mental Health. 